\title{
Attenuated Directed Exploration during Reinforcement Learning in Gambling Disorder
}

\author{
${ }^{\circledR}$ A. Wiehler, ${ }^{1,2,3}$ K. Chakroun, ${ }^{1}$ and ${ }^{\circledR}$ J. Peters ${ }^{1,4}$ \\ ${ }^{1}$ Department of Systems Neuroscience, University Medical Center Hamburg-Eppendorf, Hamburg 20246, Germany, ${ }^{2}$ Université de Paris, Paris \\ F-75006, France, ${ }^{3}$ Department of Psychiatry, Service Hospitalo-Universitaire, Groupe Hospitalier Universitaire Paris Psychiatrie \& Neurosciences, \\ Paris F-75014, France, and ${ }^{4}$ Department of Psychology, Biological Psychology, University of Cologne, Cologne 50923, Germany
}

Gambling disorder (GD) is a behavioral addiction associated with impairments in value-based decision-making and behavioral flexibility and might be linked to changes in the dopamine system. Maximizing long-term rewards requires a flexible tradeoff between the exploitation of known options and the exploration of novel options for information gain. This explorationexploitation trade-off is thought to depend on dopamine neurotransmission. We hypothesized that human gamblers would show a reduction in directed (uncertainty-based) exploration, accompanied by changes in brain activity in a fronto-parietal exploration-related network. Twenty-three frequent, non-treatment seeking gamblers and twenty-three healthy matched controls (all male) performed a four-armed bandit task during functional magnetic resonance imaging (fMRI). Computational modeling using hierarchical Bayesian parameter estimation revealed signatures of directed exploration, random exploration, and perseveration in both groups. Gamblers showed a reduction in directed exploration, whereas random exploration and perseveration were similar between groups. Neuroimaging revealed no evidence for group differences in neural representations of basic task variables (expected value, prediction errors). Our hypothesis of reduced frontal pole (FP) recruitment in gamblers was not supported. Exploratory analyses showed that during directed exploration, gamblers showed reduced parietal cortex and substantia-nigra/ventral-tegmental-area activity. Cross-validated classification analyses revealed that connectivity in an exploration-related network was predictive of group status, suggesting that connectivity patterns might be more predictive of problem gambling than univariate effects. Findings reveal specific reductions of strategic exploration in gamblers that might be linked to altered processing in a fronto-parietal network and/or changes in dopamine neurotransmission implicated in GD.

Key words: exploration-exploitation; fMRI; gambling disorder; perseveration; reinforcement learning; reward

\section{Significance Statement}

Wiehler et al. (2021) report that gamblers rely less on the strategic exploration of unknown, but potentially better rewards during reward learning. This is reflected in a related network of brain activity. Parameters of this network can be used to predict the presence of problem gambling behavior in participants.

\section{Introduction}

Gambling disorder (GD) has a lifetime prevalence of around $1 \%$ (Kessler et al., 2008; Lorains et al., 2011). In the DSM-5, it is classified in the category of substance use and addictive disorders, reflecting the considerable overlap in behavioral and neural

Received June 26, 2020; revised Jan. 18, 2021; accepted Jan. 22, 2021.

Author contributions: A.W. and J.P. designed research; A.W. performed research; A.W. contributed unpublished reagents/analytic tools; A.W. and K.C. analyzed data; A.W. and J.P. wrote the paper.

This work was supported by the Deutsche Forschungsgemeinschaft Grant PE1627/5-1 (to J.P.). We thank Anica Bäuning for assistance with task programming and Raymond Dolan and Nathaniel Daw for kindly making the behavioral data from their 2006 paper available for re-analysis.

The authors declare no competing financial interests.

Correspondence should be addressed to A. Wiehler antonius.wiehler@gmail.com or J. Peters jan.peters@ uni-koeln.de.

https://doi.org/10.1523/JNEUROSCI.1607-20.2021

Copyright $\odot 2021$ the authors correlates with substance-based addictions (Goudriaan et al., 2019). For example, activity in reward-related brain regions, including the ventral striatum (VS) and medial prefrontal cortex (mPFC), has repeatedly been found to differ between healthy controls and participants with GD (Balodis et al., 2012; Leyton and Vezina, 2012; Miedl et al., 2012), although with inconsistent directionality (Clark et al., 2019).

In addition to increased temporal discounting and risk-taking (Wiehler and Peters, 2015), gamblers also exhibit cognitive impairments reflected in reduced behavioral flexibility. This includes impaired performance in the Stroop task and increased perseveration following rule changes in the Wisconsin Card Sorting Task (van Timmeren et al., 2018). State-dependent modulations of risk-attitude have been found impaired in problem gambling (Fujimoto et al., 2017). Similar impairments are observed in reversal learning, where gamblers make more 
perseveration errors following contingency reversals (de Ruiter et al., 2009; Boog et al., 2014), an effect that has been linked to maladaptive control beliefs about gambling outcomes, which might interfere with decision-making (Lim et al., 2015).

More generally, reward-learning entails a trade-off between exploitation of options with known value, and exploration of novel options for information gain (Wilson et al., 2021). One of the most widely used tasks to examine exploration behavior is the multi-armed-bandit task (Daw et al., 2006). Here, participants make repeated choices between multiple choice options ("bandits") to obtain rewards. Exploitation involves tracking each bandit's expected value and choosing the best. In contrast, exploration can be undirected because of stochastic selection of bandits ("random exploration"; Daw et al., 2006; Schulz and Gershman, 2019). Additionally, exploration might entail a goal-directed component and depend on the bandit's estimated uncertainty ("directed exploration"; Speekenbrink and Konstantinidis, 2015; Schulz and Gershman, 2019; Chakroun et al., 2020).

A bilateral fronto-parietal network supports exploration, including intra-parietal sulcus and fronto-polar cortex (Daw et al., 2006; Raja Beharelle et al., 2015; Chakroun et al., 2020). Although initially characterized in the context of random exploration (Daw et al., 2006), fronto-polar cortex may more specifically support directed exploration (Boorman et al., 2009, 2011; Badre et al., 2012; Zajkowski et al., 2017).

There is substantial evidence implicating the neurotransmitter dopamine (DA) in the pathophysiology of GD (Kayser, 2019). Likewise, a contribution of DA to the regulation of the exploration-exploitation trade-off is suggested both by theory (Beeler, 2012) and empirical data (Frank et al., 2009; Kayser et al., 2015; Gershman and Tzovaras, 2018; Cinotti et al., 2019; Chakroun et al., 2020). The most prominent empirical observation implicating DA in gambling comes from patients suffering from Parkinson's disease, where higher rates of problem gambling behavior haven been liked to pharmacological DA replacement therapy (Driver-Dunckley et al., 2003; Voon et al., 2006). Gamblers may also exhibit increased presynaptic striatal DA levels (Boileau et al., 2014; van Holst et al., 2018), although this is controversially discussed (Majuri et al., 2017; Potenza, 2018).

We have recently shown that an elevation of DA levels via LDopa attenuates directed exploration in healthy controls (Chakroun et al., 2020). If one conceptualizes GD as a hyperdopaminergic state (Boileau et al., 2014; van Holst et al., 2018), this entails the prediction that GD might likewise be associated with reduced directed exploration. This hypothesis resonates with the discussed impairments in behavioral flexibility in GD. In line with the critical role of frontal pole (FP) regions (Daw et al., 2006; Raja Beharelle et al., 2015; Zajkowski et al., 2017) and prefrontal dopamine (Frank et al., 2009) in exploration, we further hypothesized that reduced FP recruitment might contribute to reduced exploration in GD. We addressed these hypotheses in a group of frequent gamblers (with sixteen out of twenty-three meeting the diagnostic criteria for GD) and healthy matched controls using an established four-armed bandit task during functional magnetic resonance imaging (fMRI; Daw et al., 2006).

\section{Materials and Methods}

Sample

We investigated a sample of $n=23$ frequent gamblers [age mean (SD) $=$ 25.91 (6.47), all male]. Sixteen gamblers fulfilled four or more DSM-5 criteria of gambling disorder [mean $(\mathrm{SD})=6.31(1.45)$, previously defined as pathologic gamblers]. Seven gamblers fulfilled one to three criteria [mean $(\mathrm{SD})=2.43(0.77)$, previously defined as problem
Table 1. Summary of demographics and group matching statistics

\begin{tabular}{|c|c|c|c|c|c|c|c|}
\hline & \multicolumn{2}{|c|}{$\begin{array}{l}\text { Gamblers } \\
(n=23)\end{array}$} & \multicolumn{2}{|l|}{$\begin{array}{l}\text { Controls } \\
(n=23)\end{array}$} & \multirow[b]{2}{*}{$t$} & \multirow[b]{2}{*}{ df } & \multirow[b]{2}{*}{$p$} \\
\hline & Mean & SD & Mean & SD & & & \\
\hline Age & 25.91 & 6.47 & 26.52 & 5.92 & -0.33 & 43.65 & 0.74 \\
\hline School years & 11.64 & 1.77 & 11.91 & 1.35 & -0.60 & 41.04 & 0.55 \\
\hline Monthly income in Euros & 1439.86 & 835.84 & 1093.76 & 460.07 & 1.72 & 34.81 & 0.09 \\
\hline FTND & 2.14 & 2.58 & 1.83 & 2.15 & 0.44 & 42.58 & 0.66 \\
\hline AUDIT & 6.09 & 7.14 & 6.52 & 4.57 & -0.24 & 37.44 & 0.81 \\
\hline DSM-5 score & 5.13 & 2.22 & 0.09 & 0.29 & 10.80 & 22.74 & $<0.01$ \\
\hline KFG & 25.91 & 14.15 & 0.48 & 1.12 & 8.59 & 22.28 & $<0.01$ \\
\hline SOGS & 8.64 & 4.46 & 0.22 & 0.52 & 9.00 & 22.60 & $<0.01$ \\
\hline BDI-II & 15.41 & 11.41 & 7.61 & 7.94 & 2.69 & 39.27 & 0.01 \\
\hline
\end{tabular}

FTND, Fagerström test of nicotine dependence; AUDIT, alcohol use disorders identification test; KFG, Kurzfragebogen zum glücksspielverhalten; SOGS, South Oaks gambling screen; BDI-II: Beck depression inventory-II.

gamblers]. All participants reported no other addiction except for nicotine. Current drug abstinence was verified via urine drug screening. All participants reported no history of other psychiatric or neurologic diagnoses except depression. No participant was undergoing any psychiatric treatment. Current psychopathology was controlled using the Symptom Checklist 90 Revised (SCL-90-R) questionnaire (Schmitz et al., 2000) and depression symptoms were assessed via the Beck Depression Inventory-II (BDI-II, Osman et al., 2004). To characterize gambling behavior, we conducted the German gambling questionnaire Kurzfragebogen zum Glücksspielverhalten (KFG; Petry, 1996), the German version of the South Oaks Gambling Screen (SOGS, Lesieur and Blume, 1987) and the Gambling Related Cognitions Scale (GRCS; Raylu and Oei, 2004). Participants were recruited via advertisements placed on local Internet boards but were not searching for treatment.

We recruited $n=23$ healthy control participants, matched for age, gender, education, income, alcohol [Alcohol Use Disorders Identification Test (AUDIT); Saunders et al., 1993], and nicotine consumption [Fagerström Test of Nicotine Dependence (FTND); Heatherton et al., 1991; see Table 1]. Four of these control participants were included from an earlier study that used the exact same task and imaging protocol (Chakroun et al., 2020). To rule out drug or order effects, we included the first imaging session of participants who completed the placebo condition first. Furthermore, these four participants were selected to maximize matching to the gamblers group in terms of age, education and income. All results were significant without these four additional participants.

All participants provided informed written consent before participation and the study procedure was approved by the local institutional review board (Hamburg Board of Physicians).

\section{Task and procedure}

Participants completed two sessions of testing on separate days. The first session included all questionnaires and an assessment of the spontaneous eye-blink rate, that was published previously (Mathar et al., 2018). The second session started with a training session of the task, followed by fMRI and structural MRI. Subsequently, they performed an additional task in the MRI that will be reported elsewhere.

We used a previously described four-armed bandit task (Daw et al., 2006). We applied the same task as in the original publication, with the exception that we replaced slot machine images for each bandit with colored boxes (Fig. 1A). On each trial, participants selected one of four bandits. They received a payout between 0 and 100 points for the chosen bandit, which was added to a total score. The points that could be won on each trial were determined by Gaussian random walks, leading to payouts fluctuating slowly throughout the experiment (Fig. 1B; for mathematical details, see below). Participants completed 300 trials in total that were split into four blocks separated by short breaks. We instructed participants to gain as many points as possible during the experiment. Reimbursement was a fixed baseline amount plus a bonus that depended on the number of points won in the bandit task. In total, participants received between 70 and 100 Euros for participation. 
A

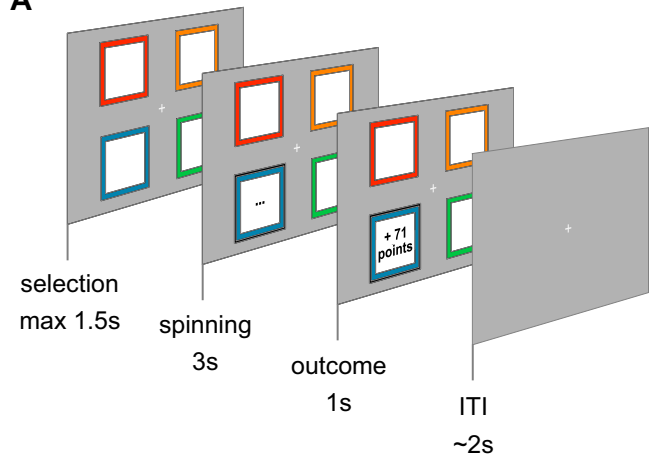

B

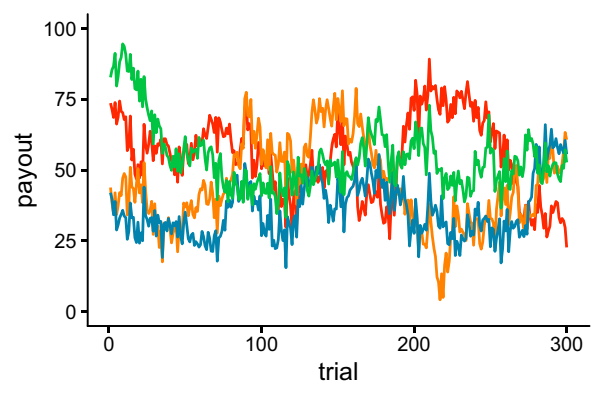

Figure 1. Task. $\boldsymbol{A}$, One trial of the bandit task. On each trial, participants choose between four bandits on the screen and received a payout in reward points. $\boldsymbol{B}$, Payouts fluctuated across the 300 trials of the experiment according to Gaussian random walks. Here, one example set of random walks is shown. Colors correspond to bandits in $\boldsymbol{A}$.

\section{Computational modeling}

To quantify exploration behavior, participants' choices were fitted with several reinforcement learning models of varying complexity. We first implemented a Q-learning model (Sutton and Barto, 1998). Here, participants update the expected value (Q-value) of the ith bandit on trial $t$ via a prediction error $\delta_{t}$ :

$$
Q_{i, t+1}=Q_{i, t}+\alpha \delta_{t}
$$

with

$$
\delta_{t}=r_{t}-Q_{i, t}
$$

Here, $Q$ is the expected value of the $i$ th bandit on trial $t, \alpha$ is a constant learning rate, that determines the proportion of the prediction error $\delta_{t}$ that is used for the value update, and $r_{t}$ is the reward outcome on trial $t$. In this model unchosen bandits are not updated but retain their previous $Q$ values.

$Q$ values are transformed into action probabilities, using a softmax choice rule:

$$
p\left(c_{t}=i\right)=\frac{\exp \left(\beta Q_{i, t}\right)}{\sum_{j} \exp \left(\beta Q_{j, t}\right)} .
$$

Here, $p$ is the probability of choice $c_{t}$ of bandit $i$ in trial $t$, given the estimated values $Q$ from Equation 1 for all $j$ bandits. $\beta$ denotes an inverse temperature parameter, that models choice stochasticity: for greater values of $\beta$, choices become more dependent on the learned $Q$ values. Conversely, as $\beta$ approaches 0 , choices become more random. In this model, $\beta$ controls the exploration-exploitation trade-off such that for higher values of $\beta$, exploitation dominates, whereas exploration increases as $\beta$ approaches 0 . Note, however, that this model does not incorporate uncertainty about $Q$ values, as only mean $Q$ values are tracked.

We next examined a Bayesian learner model (Kalman filter) that was also applied by Daw et al. (2006). This model assumes that participants use a representation of the Gaussian random walks that constitute the task's payout structure. Thus, regardless of the choice, mean and variance of each bandit $i$ are updated on each trial $t$ as follows:

$$
\begin{gathered}
\hat{\mu}_{i, t+1}=\lambda \hat{\mu}_{i, t}+(1-\lambda) \theta \\
\hat{\sigma}_{i, t+1}^{2}=\lambda^{2} \hat{\sigma}_{i, t}^{2}+\sigma_{d}^{2} .
\end{gathered}
$$

Here, $\mu$ is the mean expected value, $\sigma$ is the SD of the expected value, $\lambda$ is a decay rate (fixed to 0.9836), $\theta$ is the decay center (fixed to 50), and $\sigma_{d}$ is the SD of the diffusion noise (fixed to 2.8). Note that these equations are used to generate the Gaussian walks (see Daw et al., 2006). That is, without sampling, each bandits' mean value slowly decayed toward $\theta$, and SDs increased $\sigma_{d}$ units per trial.
The bandit chosen on trial $t\left(c_{t}\right)$ is additionally updated using a $\delta$ rule similar to Equation 2:

$$
\hat{\mu}_{c_{t}, t+1}=\hat{\mu}_{c_{t}, t}+k_{t} \delta_{t}
$$

with

$$
\delta_{t}=r_{t}-\hat{\mu}_{c_{t}, t}
$$

and

$$
k_{t}=\frac{\hat{\sigma}_{c_{t} t}^{2}}{\hat{\sigma}_{c_{t}, t}^{2}+\sigma_{o}^{2}} .
$$

Equation 6 is analogous to Equation 1, with one important exception: while the Q-learning model assumes that the learning rate is constant, in the Kalman filter model, the learning rate is uncertainty-dependent. The trial-wise learning rate $k_{t}$ (Kalman gain) depends on the current estimate of the uncertainty of the bandit that is sampled (as per Eq. 8) such that the mean expected value is updated more when bandits with higher uncertainty are sampled. Specifically, $\hat{\sigma}_{c t, t}$ refers to the estimated uncertainty of the expected value of the chosen bandit, and $\sigma_{o}$ is the observation $\mathrm{SD}$, that is, the variance of the normal distribution from which payouts are drawn (fixed to 4). The uncertainty of the expected value of the chosen bandit is then updated according to

$$
\hat{\sigma}_{c_{t}, t+1}^{2}=\left(1-k_{t}\right) \hat{\sigma}_{c_{t}, t}^{2}
$$

Taken together, this model gives rise to the following intuitions. First, participants not only track the expected mean payoff $(\mu)$ but also the uncertainty about the expected mean payoff $(\sigma)$. The mean expected value of unsampled bandits is gradually moving toward the decay center and uncertainty about the value increases. Sampling of a bandit leads to a reduction in uncertainty (Eq. 9) that is proportional to the uncertainty before sampling. Additionally, the bandit's mean value is updated via the prediction error (Eq. 7) weighted by the trialwise learning rate (Eq. 8) such that updating is substantially higher when sampling from uncertain bandits.

We next combined this algorithm for value updating with three different choice rules for action selection. First, we used a standard softmax model (see Eq. 3). Here, choices are only based on the mean value estimates of the bandits $\mu_{i, t}$, such that exploration occurs in inverse proportion to the softmax parameter $\beta$ and the differences in value estimates:

$$
p\left(c_{t}=i\right)=\frac{\exp \left(\beta \hat{\mu}_{i, t}\right)}{\sum_{j} \exp \left(\beta \hat{\mu}_{j, t}\right)} .
$$

Second, we added an "exploration bonus" parameter $\varphi$ that scales a bandit's uncertainty $\hat{\sigma}_{i, t}$ and adds this scaled uncertainty as a value 
bonus for each bandit, as first described by Daw et al. (2006). This term implements directed exploration so that choices are specifically biased toward uncertain bandits.

$$
p\left(c_{t}=i\right)=\frac{\exp \left(\beta\left[\hat{\mu}_{i, t}+\varphi \hat{\sigma}_{i, t}\right]\right)}{\sum_{j} \exp \left(\beta\left[\hat{\mu}_{j, t}+\varphi \hat{\sigma}_{j, t}\right]\right)} .
$$

Following a similar logic, we next included a parameter $\rho$ modeling choice perseveration. $\rho$ models a value bonus for the bandit chosen on the previous trial:

$$
\begin{gathered}
\mathbf{1}_{c_{t-1}}(i):=\left\{\begin{array}{l}
1 \text { if } i=c_{t-1} \\
0 \text { if } i \neq c_{t-1}
\end{array}\right. \\
p\left(c_{t}=i\right)=\frac{\exp \left(\beta\left[\hat{\mu}_{i, t}+\rho \mathbf{1}_{c_{t-1}}(i)\right]\right)}{\sum_{j} \exp \left(\beta\left[\hat{\mu}_{j, t}+\rho \mathbf{1}_{c_{t-1}}(j)\right]\right)} .
\end{gathered}
$$

Finally, we set up a full model including both directed exploration $(\varphi)$ and perseveration $(\rho)$ terms:

$$
p\left(c_{t}=i\right)=\frac{\exp \left(\beta\left[\hat{\mu}_{i, t}+\varphi \hat{\boldsymbol{\sigma}}_{i, t}+\rho \mathbf{1}_{c_{t-1}}(i)\right]\right)}{\sum_{j} \exp \left(\beta\left[\hat{\mu}_{j, t}+\varphi \hat{\sigma}_{j, t}+\rho \mathbf{1}_{c_{t-1}}(j)\right]\right.} .
$$

In total, our model space therefore consisted of five models: (1) Qlearning model with softmax, (2) Bayesian learner with softmax, (3) Bayesian learner with softmax and exploration bonus, (4) Bayesian learner with softmax and perseveration bonus, and (5) Bayesian learner with softmax, exploration bonus and perseveration bonus. All models were fitted using hierarchical Bayesian parameter estimation in Stan version 2.18.1 (Carpenter et al., 2017) with separate group-level normal distributions for gamblers and controls for each choice parameter $(\beta, \varphi$, and $\rho$ ), from which individual-participant parameters were drawn. We ran four chains with $5 \mathrm{k}$ warmup samples and retained 10k samples for analysis. Group-level priors for means were set to uniform distributions over sensible ranges $(\beta=[0,3] ; \varphi=[-20,20] ; \rho=[-20,20])$. Group level priors for variance parameters were set to half-Cauchy with mode 0 and scale 3 .

To verify that group differences in the choice parameters $(\beta, \varphi$, and $\rho$ ) were not confounded by group differences in the walk parameters, we estimated supplementary models were the random walk parameters were allowed to vary. Parameters were estimated one at a time because of convergence issues, and in a non-hierarchical fashion (i.e., one parameter per group) with the following uniform priors: $\lambda=[0,1] ; \theta=[0,100]$, $\sigma_{d}=[0,20]$.

Model comparison was performed using the Watanabe-Akaike Information Criterion (WAIC; Watanabe, 2010; Vehtari et al., 2017) where smaller values indicate a better fit. To examine group differences in the parameters of interest $(\beta, \varphi$, and $\rho)$ we examined the posterior distributions of the group-level parameter means. Specifically, we report mean posterior group differences, standardized effect sizes for group differences and Bayes factors testing for directional effects (Marsman and Wagenmakers, 2017; Pedersen et al., 2017). Directional Bayes factors (dBFs) were computed as $d B F=i / 1-i$ where $i$ is the integral of the posterior distribution of the group difference from 0 to $+\infty$, which we estimated via non-parametric density estimation.

\section{fMRI setup}

MRI data were collected with a Siemens Trio 3T system using a 32-channel head coil. fMRI was recorded in four blocks. Each volume consisted of 40 slices $(2 \times 2 \times 2 \mathrm{~mm}$ in-plane resolution and $1 \mathrm{~mm}$ gap, repetition time $=2.47 \mathrm{~s}$, echo time $26 \mathrm{~ms}$ ). We tilted volumes by $30^{\circ}$ from the anterior and posterior commissures connection line to avoid distortions in the frontal cortex (Deichmann et al., 2003). Participants viewed the screen via a head-coil mounted mirror. High-resolution T1 and MT weighted structural images were acquired after functional scanning was completed.

\section{fMRI preprocessing}

MRI data preprocessing and analysis was done using SPM12 (Wellcome Department of Cognitive Neurology, London, United Kingdom). First, volumes were realigned and unwarped to account for head movement and distortion during scanning. Second, slice time correction to the onset of the middle slice was performed to account for the shifted acquisition time of slices within a volume. Third, structural images were coregistered to the functional images. Finally, all images were smoothed (8 mm FWHM) and normalized to MNI-space using DARTEL tools and the VBM8 template.

\section{fMRI analysis}

On the first level, we used general linear models (GLMs) implemented in SPM12. GLM 1 included the following regressors: (1) trial onset, (2) trial onset modulated by a binary parametric modulator coding whether the trial was a random exploration trial, (3) trial onset modulated by a binary parametric modulator coding whether the trial was a directed exploration trial, (4) outcome onset, (5) outcome onset modulated by model-based prediction error, and (6) outcome onset modulated by model-based expected value of the chosen bandit. Missing responses were modeled separately.

Based on the best-fitting computational model, trials were classified. Exploitation trials are trials with choices of the bandit with the highest sum of expected value, uncertainty bonus and perseveration bonus (i.e., the highest softmax probability). Exploration trials are all other trials. These were further subdivided into trials on which participants selected the bandit with the highest exploration bonus (directed exploration trials) and all other trials (random exploration trials). Please note that the trial classification in GLM 1 leads to exploitation trials to be the baseline and exploration as activation relative to this baseline.

GLM 2 included the following regressors: (1) trial onset, (2) outcome onset, and (3) outcome onset modulated by the number of points earned. Missing responses were modeled separately.

GLM 3 is following GLM 1 but replaced the trial classification by the summed uncertainty of all four choice options. Thus, it included the following regressors: (1) trial onset, (2) trial onset modulated by the summed uncertainty of all choice options, (3) outcome onset, (4) outcome onset modulated by model-based prediction error, and (5) outcome onset modulated by the model-based expected value of the chosen bandit. Missing responses were modeled separately.

Group differences were assessed by a second-level random-effects analysis (two-sample $t$ test). Here, we included covariates for depression (BDI-II score), alcohol consumption (AUDIT score), smoking (FTND score), and age. Covariates were $z$-scored across both groups.

\section{Dynamic causal modeling (DCM)}

DCM (Stephan et al., 2008) is a method to formally test and compare different causal connectivity models underlying the BOLD signal. First, we extracted the BOLD time course of regions of interest (ROIs). Following our previous approach (Chakroun et al., 2020), we defined four ROIs of the right hemisphere based on previous research (Daw et al., 2006; Blanchard and Gershman, 2018): Frontal pole (FP), intraparietal sulcus (IPS), anterior insula (aIns), and dorsal anterior cingulate cortex (dAcc; for coordinates, see Table 2). Time courses were extracted from 5-mm spheres around the single-participant peak within the ROI. See Results for more details on the tested models.

\section{Classification analysis}

To examine whether connectivity dynamics in an exploration-related network was associated with group status (see the previous paragraph), we used an unbiased, leave-one-pair-out approach for group membership classification. We trained a support vector machine (SVM) classifier (Chang and Lin, 2011; C =1) on all participants except one patient and one control. The prediction accuracy was computed based on the leftout pair. We repeated this for all possible pairs of controls and gamblers and averaged accuracies across left-out pairs. Finally, we repeated this procedure 500 times with randomly shuffled labels to build a null-distribution, which allows assessing the significance of the observed accuracy. 
Table 2. ROI analysis

\begin{tabular}{|c|c|c|c|c|}
\hline Reference & Location & Coordinates $(x / y / z)$ & $\begin{array}{l}\text { Main effect } \\
\text { Directed exploration }>\text { exploitation }\end{array}$ & $\begin{array}{l}\text { Controls }>\text { Gamblers } \\
\text { Directed exploration }>\text { exploitation }\end{array}$ \\
\hline Daw et al. (2006) & R FP & $27,57,6$ & $p=0.012$ & No cluster \\
\hline Daw et al. (2006) & L FP & $-28,48,4$ & $p<0.001$ & No cluster \\
\hline Daw et al. (2006) & R IPS & $39,-36,42$ & $p<0.001$ & $p=0.223$ \\
\hline Daw et al. (2006) & L IPS & $-29,-33,45$ & $p<0.001$ & No cluster \\
\hline Blanchard and Gershman (2018) & $\mathrm{R}$ alns & $32,22,-8$ & $p=0.003$ & $p=0.14$ \\
\hline Blanchard and Gershman (2018) & L alns & $-30,16,-8$ & $p=0.002$ & $p=0.05$ \\
\hline Blanchard and Gershman (2018) & $\mathrm{R} d A C \mathrm{C}$ & $8,16,46$ & $p<0.001$ & $p=0.048$ \\
\hline
\end{tabular}

Ten-millimeter spheres were placed around the coordinates of exploration related activations of previous studies; $p$ values are small volume corrected. See also Chakroun et al. (2020; their Appendix 1 and Table 5).

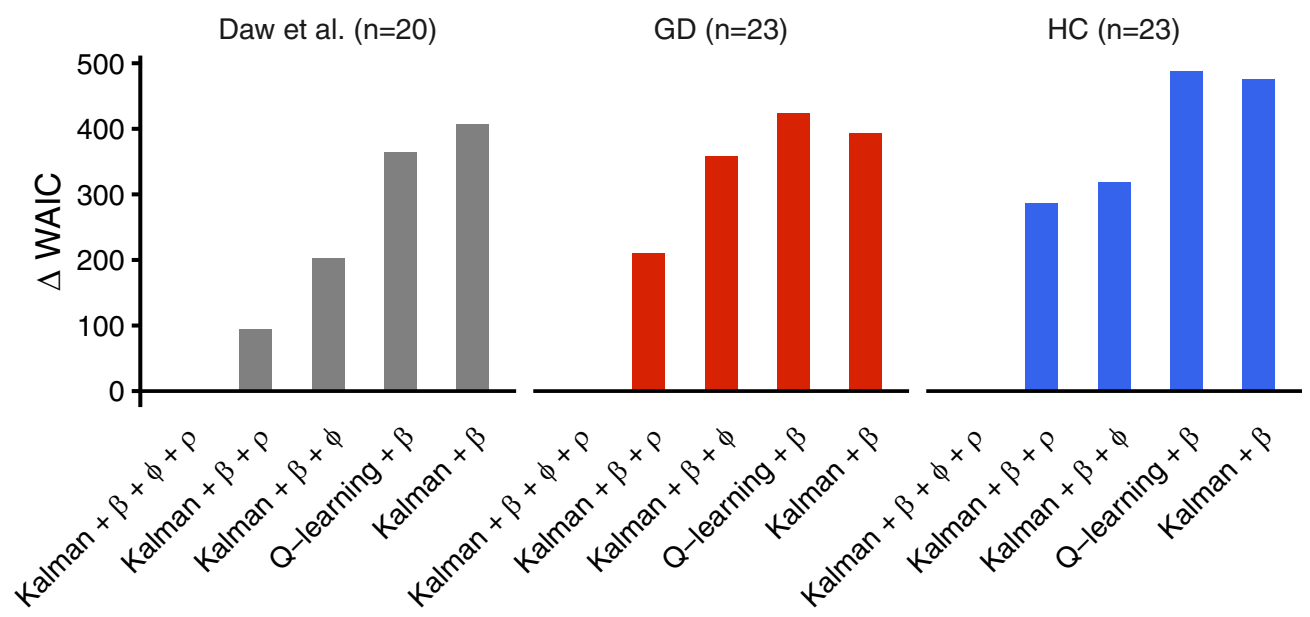

Figure 2. Results of the model comparison based on the WAIC. Plotted are WAIC differences between each model and the best-fitting model, such that smaller values indicate a superior fit. Across a re-analysis of behavioral data from Daw et al. (2006; $n=20)$, gamblers (GD, $n=23)$, and matched healthy controls (HC; $n=23)$ a Kalman filter model with uncertainty bonus $(\varphi)$ and perseveration bonus $(\rho)$ accounted for the data best.

Data availability statement

The data that support the findings of this study are available on Zenodo (long format matrix for the behavioral data and t-maps for the fMRI data at https://doi.org/10.5281/zenodo.4271604).

\section{Results}

\section{Model-free results}

The group difference in the number of points earned was not significant [controls mean $(S D)=18,204.83$ (1435.37), gamblers mean $\left.(\mathrm{SD})=18,489.69(1520.32), t_{(43.86)}=-0.54, p=0.51\right]$. Median response times tended to be shorter in gamblers [controls mean $(\mathrm{SD})=0.44 \mathrm{~s}(0.05)$, gamblers mean $(\mathrm{SD})=0.40 \mathrm{~s}$ (0.07), $\left.t_{(39.413)}=1.59, p=0.11\right]$. As a model-free measure of exploration, we computed the sequential exploration index (Ligneul, 2019). This index tracks whether in each quadruple of trials all choices are unique, which might reflect a systematic exploration of options. Although this index was numerically higher in controls versus gamblers [controls mean $(\mathrm{SD})=0.065(0.067)$, gamblers mean $(\mathrm{SD})=0.057(0.034)]$, the difference was not statistically significant $\left(t_{(33)}=-0.48, p=0.6\right)$.

\section{Model comparison}

Next, we used model comparison based on the WAIC (where lower values indicate a better fit; Gelman et al., 2014) to examine the behavioral data for signatures of directed exploration and perseveration. In both groups, the Bayesian learning model (Kalman filter) with softmax, exploration bonus, and perseveration bonus accounted for the data best (Fig. 2). This model ranking was also observed when the original behavioral data from the Daw et al. (2006) study was re-analyzed using our hierarchical Bayesian estimation approach (Fig. 2).

The best-fitting model gives rise to the following intuitions. First, participants not only track the expected mean payoff $(\mu)$ but also the uncertainty about the expected mean payoff $(\sigma)$ of the four bandits. The mean expected value of unsampled bandits is gradually moving toward a decay center and uncertainty about the mean value increases. Sampling of a bandit leads to a reduction in uncertainty that is proportional to the uncertainty before sampling. Additionally, the bandit's mean value is updated via a prediction error weighted by a trial-wise learning rate (Kalman gain $\kappa$ ) such that sampling from uncertain bandits leads to more substantial updating compared with sampling from a bandit with lower uncertainty. Second, action selection is then a function of the mean expected value of the bandits, an uncertainty bonus (which favors selecting bandits which high uncertainty) and a perseveration bonus (which favors repeating the choice made on the previous trial).

\section{Parameters of the best-fitting model}

Next, we analyzed the parameters of the best-fitting model in detail, focusing on choice stochasticity (softmax slope $\beta$ ), exploration bonus (directed, uncertainty-based exploration $\varphi$ ), and perseveration bonus ( $\rho$; see Fig. 3 ). There was evidence for a decrease in $\varphi$ in the gamblers (Fig. 3D), such that a decrease in directed exploration in gamblers was $\sim 12$ times more likely than an increase, given the data $(\mathrm{dBF}=12.15)$. Choice stochasticity $\beta$ 

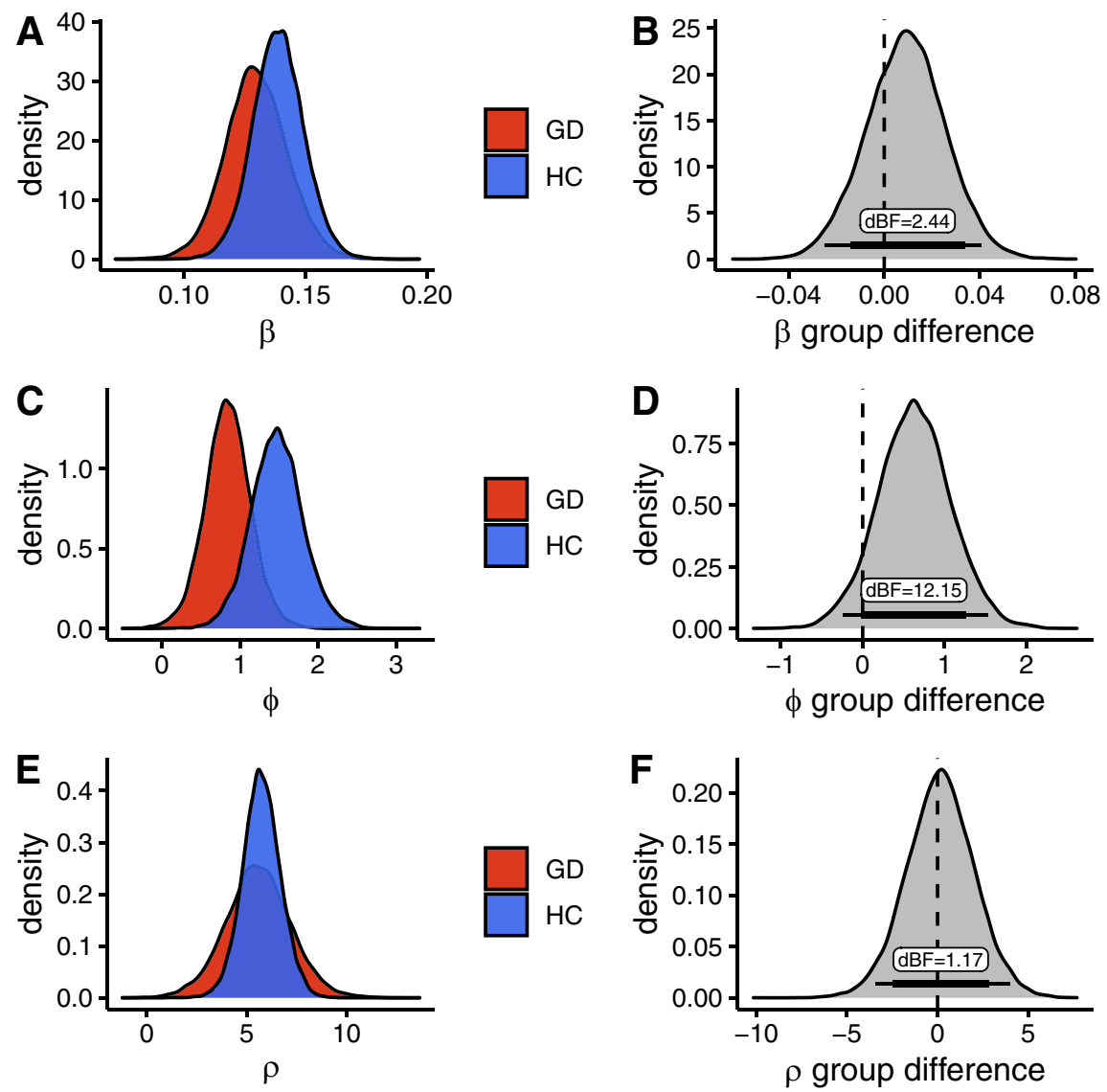

Figure 3. Group parameters of the best-fitting model. GD, gambling disorder; $\mathrm{HC}$, healthy controls. $\boldsymbol{A}, \boldsymbol{C}, \boldsymbol{E}$, Posterior distribution of group-level parameters per group. $\boldsymbol{B}, \boldsymbol{D}, \boldsymbol{F}$, Distribution of differences in posterior distributions between groups. Bottom lines indicate the $85 \%$ and $95 \%$ highest density interval of the distribution. $\mathrm{dBF}$ : directed Bayes factor, the proportion of the difference distribution above 0 over the proportion of the difference distribution below $0 . \boldsymbol{A}, \boldsymbol{B}, \beta$ parameter, which represents random exploration. C, D, $\varphi$ parameter which represents directed exploration. $\boldsymbol{E}, \mathbf{F}, \rho$ parameter which represents perseveration.

and perseveration $\rho$, on the other hand, were similar between groups such that the group difference distributions were in each case centered at zero and of inconclusive directionality $(\mathrm{dBF}=2.44$ and $\mathrm{dBF}=1.17$; see Fig. $3 B, F)$.

As an additional test of whether groups were statistically distinguishable based on this model, we re-fit the best fitting model with single group-level Gaussian distributions per parameter (as opposed to modeling separate group-level gaussians for each group). A model comparison between this model with group-level distributions shared between groups and the original model with separate group-level parameters for controls and gamblers provided further evidence for a group difference: the data were better accounted for by a model with separate versus shared

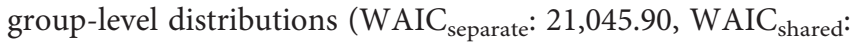
21,049.57).

We next explored whether individual differences in gambling addiction severity were associated with exploration behavior in the gamblers. As an index of addiction severity, we computed the mean $z$ score of SOGS and KFG scores. The correlation between addiction severity and single-participant $\varphi$ parameters was not significant $(r=0.01, p=0.95)$. $\varphi$ parameters also did not correlate with any sub-scale of the Gambling Related Cognition Scale (GRCS, all correlations $r<0.2, p>0.38$ ). To explore the effect of potential covariates on $\varphi$, we performed a regression analysis across both groups. We predicted $\varphi$ based on income, AUDIT score (alcohol consumption), age, BDI-II (depression), GRCS (gambling cognitions), FTND score (nicotine addiction), school years and gambling addiction severity. None of the predictors were significant.

\section{Group differences in random walk parameters}

The observed changes in $\varphi$ could have been affected by differences in the representations of the random walks between groups. Because the corresponding parameters $\left(\lambda, \theta\right.$, and $\left.\sigma_{d}\right)$ were fixed in our initial analysis, we ran additional models in which these parameters were free to vary. Because of convergence problems, these parameters were estimated one at a time, and in a non-hierarchical fashion. Still, $\sigma_{d}$ could not be estimated reliably because of convergence issues, but allowing $\theta$ to vary between groups still revealed a group difference in $\varphi$ $(\mathrm{dBF}=13.18)$, and the same was true when $\lambda$ was allowed to vary $(\mathrm{dBF}=$ 9.05). While $\theta$ was similar between groups $(\mathrm{dBF}=0.44)$, there was evidence for an increase in $\lambda$ in gamblers versus controls $(\mathrm{dBF}=0.08)$.

\section{fMRI}

Group conjunctions

We first examined standard parametric and categorical contrasts, focusing on conjunction effects testing for consistent effects across groups (GLM2). VentromPFC (vmPFC), VS, and posterior cingulate cortex (PCC) parametrically tracked outcome value, in line with numerous previous studies and meta-analyses (Daw et al., 2006; Bartra et al., 2013; Clithero and Rangel, 2014; see Fig. 4). Importantly, outcome value effects in these regions were observed across controls and gamblers, with no evidence for a group difference.

We computed model-based prediction errors for each trial based on the single-participant parameter estimates of the bestfitting model. As previously described in healthy participants (Daw et al., 2006; Pessiglione et al., 2006), the VS bilaterally coded these prediction errors in both groups (main effect FWE corrected $p<0.05$ : peak at $x=-10, y=8, z=-14, z=6.49$ and at $16,10,-14 ; z=6.11$; group conjunction $p<0.001$ uncorrected: $-14,10,-14, z=4.65 ; 16,10,-14, z=4.32$ ).

Based on the computational model, trials were classified as exploitation, directed exploration or random exploration. Figure $5 A, B$ shows the main effect of directed exploration $>$ exploitation with extensive effects in a fronto-parietal network, replicating previous findings using the same task (Daw et al., 2006; Chakroun et al., 2020). ROI analyses using the same set of ROIs as in our previous study (Chakroun et al., 2020) confirmed significant main effects of directed exploration $>$ exploitation in bilateral FP, bilateral IPS, aIns, and dAcc (10-mm spheres around the peak coordinates; see Table 2). 


\section{Group differences in exploration-related effects}

Next, we next tested our initial hypothesis of reduced FP effects during directed exploration in gamblers (GLM1). We checked for group differences within $10-\mathrm{mm}$ spheres around the peak activations of previous studies (see Table 2), which only revealed one group difference in the R dAcc $(p<0.048)$, which did not survive correction for multiple comparisons across the set of ROIs. We next performed an exploratory wholebrain analysis (at $p<0.001$ uncorrected) of group differences in brain activity during directed exploration. Controls showed greater activation in parietal cortex $(58,-34,42, z=3.65$, $p<0.001$ uncorrected) and in the substantia nigra/ventral tegmental area $(\mathrm{SN} / \mathrm{VTA},-12,-18,-10, \quad z=3.78$, $p<0.001$ uncorrected; Fig. 6). An exploration for effects of gambling severity on exploration-related brain activity in the gambling group revealed no suprathreshold effects even at an uncorrected threshold of $p<0.001$.

\section{Uncertainty-related effects}

Following our previous finding that dopamine is involved in the representation of overall uncertainty (Chakroun et al., 2020) and because of the potential involvement of dopamine in group differences, we tested for group differences in the representation of overall uncertainty, that is, the summed uncertainty over all four bandits. In light of our previous findings, we restricted this analysis to regions where we observed these effects before. A direct test at the peak coordinates of Chakroun et al. (2020) with 10 $\mathrm{mm}$ spherical ROIs revealed a main effect cluster in the dAcc $\left(-3,21,39, z=4.39, p_{\mathrm{SVC}}=0.001\right)$, but not in the anterior $(42$, $15,-6)$ or posterior $(-34,-20,8)$ insula. No group differences were observed in these three ROIs.

\section{DCM and group differences in connectivity}

To examine whether group differences in network interactions might also contribute to the observed exploration deficit in the gambling group, we used DCM. For each participant, we extracted the BOLD time-courses in four ROIs of the right hemisphere based on previous research (Daw et al., 2006; Blanchard and Gershman, 2018): FP, IPS, aIns, and dAcc (for coordinates, see Table 2).

As driving input, we used the binary regressor coding directed exploration trials versus other trials. Because we did not expect structural differences between the groups, all models included all reciprocal connections between the ROIs. We varied the position of the input, ranging from no input to an input to all four ROIs, resulting in 16 models. Bayesian model selection (Stephan et al., 2009) revealed that the model with input confined to the parietal cortex accounted for the data best (expected probability $=0.46$, exceedance probability $=0.99$; for a graphical depiction of the best-fitting model, see Fig. 7B). A separate model selection in both groups revealed the same ranking, with model 5 accounting for the data best. Further analysis then proceeded in two steps. First, we extracted single-participant coupling and input-weight parameters (using Bayesian model averaging (Penny et al., 2010) which normalizes extracted parameters by the model evidence per participant). Parameters of the parietal cortex to parietal cortex, FP to parietal cortex and FP to aIns connection showed a trend-level difference in gamblers versus controls (two-sample $t$ tests $p<0.1$, FDR corrected for multiple comparisons; Fig. 7C).

Second, we tested the hypothesis that the overall connectivity pattern contained information predictive of group (Brodersen et al., 2014). To this end, we used a SVM classifier to predict group membership based on all DCM parameters via a leave-one-pairout, group-size balanced cross-validation scheme. The observed classification accuracy of $73.2 \%$ was significantly above chance level ( $p=0.004$, permutation test). Thus, the DCM analyses confirmed that the pattern of functional network interactions contained information about group status, although several univariate analyses in these same ROIs did not reveal group differences.

\section{Discussion}

Here, we used a combination of computational modeling and fMRI to investigate exploration behavior in gamblers using a four-armed restless bandit task. Modeling revealed attenuated directed exploration in gamblers. FMRI showed no significant group differences in the representation of basic task variables such as outcome value and reward prediction errors. An exploratory analysis, however, revealed reduced activity during directed exploration in the SN/VTA in gamblers. DCM showed that coupling in an exploration-related network dissociated gamblers from controls.

In the light of previous findings of reduced behavioral flexibility in GD, we hypothesized gamblers to show a specific reduction in directed exploration. While both perseveration bonus parameter $(\rho)$ and random exploration $(\beta)$ were similar between groups, directed exploration $(\varphi)$ was substantially reduced in gamblers. Estimates of exploration can be confounded by choice perseveration (Payzan-Lenestour and Bossaerts, 2012; Wilson et al., 2014). This is particularly important in GD where increased perseveration has been reported (van Timmeren et al., 2018). We addressed this issue by extending existing models of exploration with an additional perseveration bonus term, such that final estimates of directed exploration were not confounded by potential group differences in perseveration (see also Chakroun et al., 2020 
A

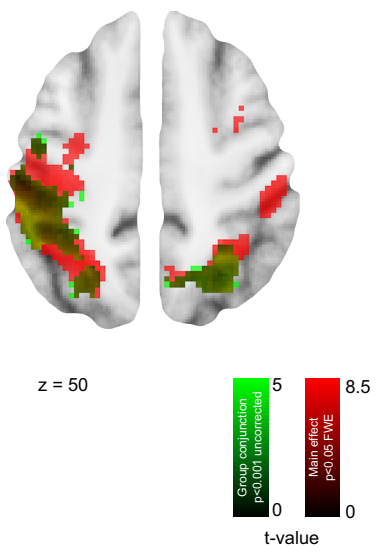

B

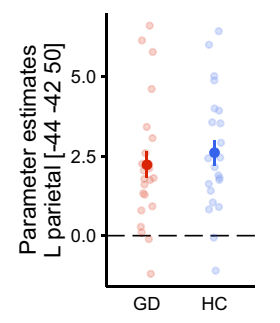

C

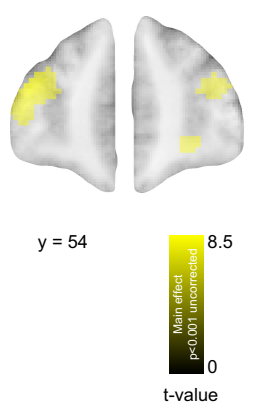

D

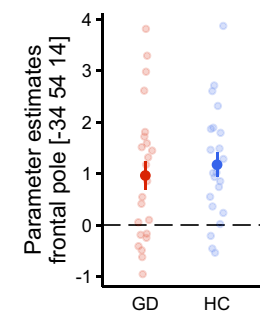

Figure 5. Neuronal correlates of directed exploration. $\boldsymbol{A}$, Contrast of directed exploration $>$ exploitation in the parietal cortex. Red, Main effect whole-brain FWE corrected $p<0.05$. Green, Conjunction $\mathrm{HC}$ and GD, $p<0.001$ (uncorrected). $\boldsymbol{B}$, Parameter estimates per participant from the left parietal cortex. $\boldsymbol{C}$, Contrast of directed exploration $>$ exploitation in the FP, main effect, $p<0.001$ (uncorrected). D, Parameter estimates per participant from the left FP. Error bars denote SEM.

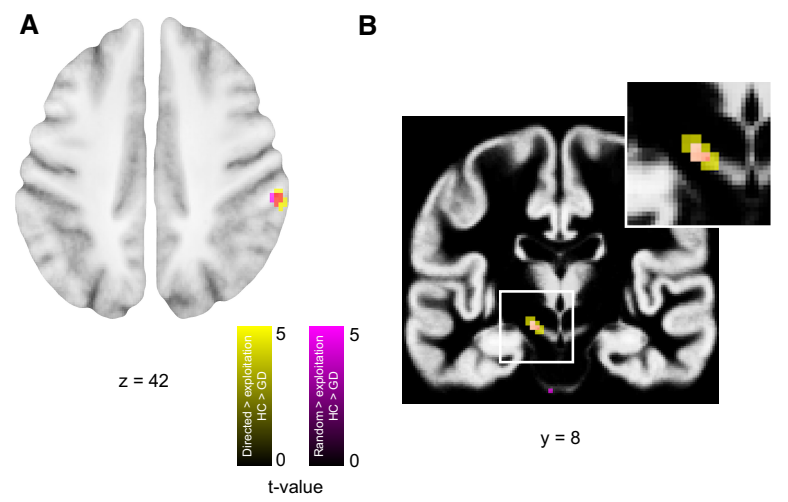

\section{C}

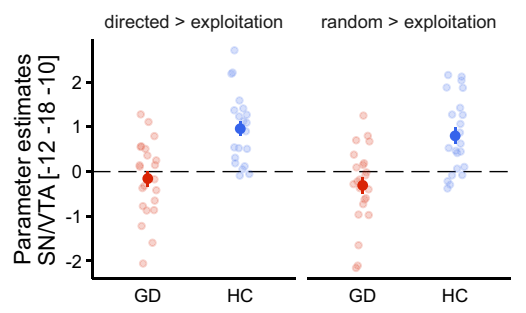

Figure 6. Group differences in the neuronal correlates of directed exploration versus exploitation. $\boldsymbol{A}$, Greater activation for controls versus gamblers in the parietal cortex, $p<0.001$ (uncorrected). $\boldsymbol{B}$, Greater activation for healthy controls compared with gamblers in the SN/VTA, $p<0.001$ (uncorrected). $\boldsymbol{C}$, Parameter estimates per participant from the peak voxel of $\boldsymbol{B}$. Note that the group difference was observed for both directed and random exploration versus exploitation. Error bars denote SEM.

for a more extensive discussion). Indeed, the full model including both directed exploration and perseveration terms accounted for the data best in both groups. This model ranking was replicated in a re-analysis of the behavioral data from Daw et al. (2006). Notably, our re-analysis of the Daw et al. (2006) behavioral data revealed a contribution of directed exploration that was not observed in their original analysis. As discussed previously (Chakroun et al., 2020), the estimation of directed exploration depends on whether perseveration is explicitly accounted for in the model. Otherwise, the model accounts for perseveration behavior by fitting a reduced (uncertainty-avoiding) exploration bonus parameter.

On the neural level, we found that basic task parameters were similarly represented in both groups. Value effects were localized in a well-characterized network including vmPFC, VS, and PCC, in line with previous meta-analysis (Bartra et al., 2013; Clithero and Rangel, 2014), with no evidence for group differences. Likewise, striatal prediction error signals were similar between groups. Again, this replicates findings in controls (McClure et al., 2003; Pessiglione et al., 2006). However, the nature of reward signals in gambling disorder addiction remains an issue of considerable debate and inconsistency (Reuter et al., 2005; Balodis et al., 2012; Leyton and Vezina, 2012; Miedl et al., 2012, 2014; van Holst et al., 2012b; Clark et al., 2019). These inconsistencies might be because of specific differences in the implementation and/or analysis of the different tasks. Our version of the fourarmed bandit task included neither gambling cues nor monetary reward cues or explicit probability information. These factors may have contributed to the null findings regarding group differences in basic parametric effects of value and prediction error. Few participants in the present sample exhibited very high levels of addiction severity (compared with Miedl et al., 2012). This might have precluded us from detecting more pronounced group differences in neural value and prediction error effects. We also did not observe correlations between gambling-related control beliefs and exploration behavior or between addiction severity and behavioral and/or fMRI readouts. While this contrasts with some previous findings using different tasks (Reuter et al., 2005; Miedl et al., 2012; van Holst et al., 2012a), overall such effects show considerable variability, both regarding behavior (Wiehler and Peters, 2015) and in reward-related imaging findings (Clark et al., 2019). Our study still included a considerable range of addiction severity (SOGS scores ranged from 3 to 17) suggesting that range restriction is an unlikely explanation for the lack of correlations. However, given the limited sample size typical of studies in such clinical populations, statistical power is an additional concern in the present study.

For the analysis of neural exploration effects, we extended previous approaches (Daw et al., 2006) by separating the neural effects of directed and random exploration via a model-based 
A

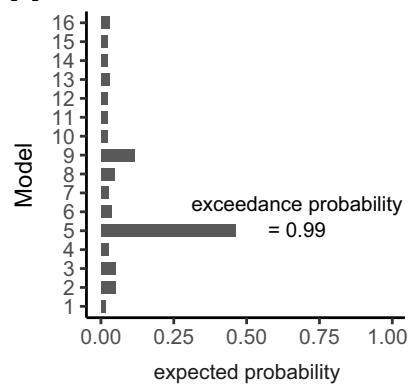

B

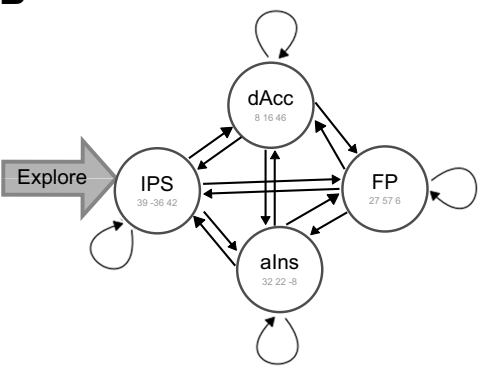

C

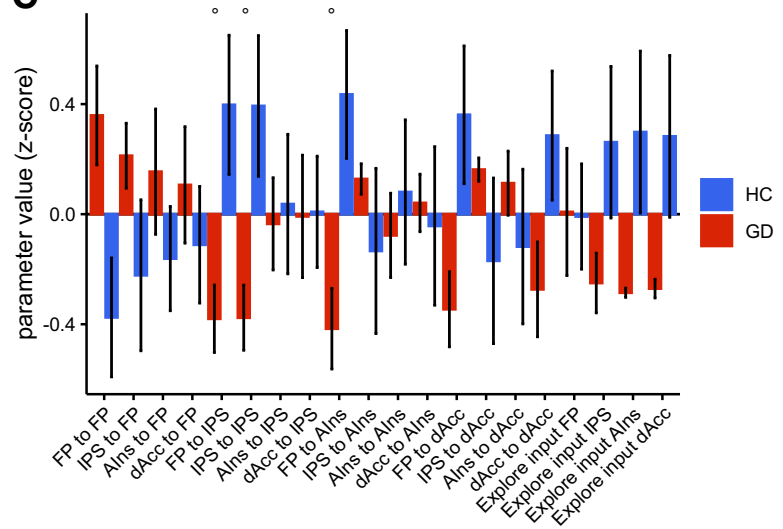

D

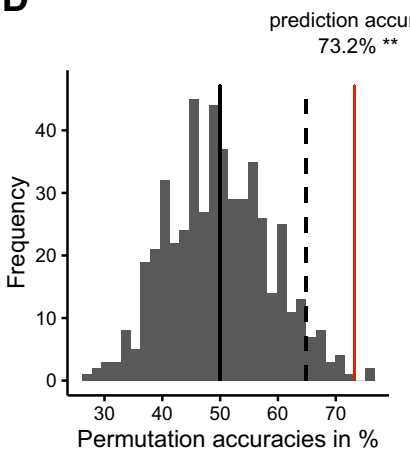

Figure 7. DCM. $\boldsymbol{A}$, Model selection of 16 models which vary all possible driving inputs. $\boldsymbol{B}$, Illustration of the DCM winning in the model selection. FP: Frontal pole, IPS: intraparietal sulcus, alns: anterior insula, dAcc: dorsal anterior cingulate cortex, Explore: Directed explore regressor as driving input in IPS. C, BMA weighted parameter comparison between groups. GD, gambling disorder; HC, healthy controls. ${ }^{\circ}$ is indicating trend-level significance ( $p<0.1$, FDR corrected). $\boldsymbol{D}$, Group identity was predicted based on the DCM parameters (leave-one-pair-out procedure). Statistical significance was assessed with a permutation test. Displayed is the distribution of prediction accuracies under the null hypothesis (500 randomly shuffled labels). The observed accuracy of $73.2 \%$ (red line) is beyond the $95 \%$ interval of the null-distribution (dashed line). Error bars denote SEM.

classification of trials. Again, overall effects were highly similar between groups and consistent with previous studies, such that directed exploration recruited a fronto-parietal network including FP regions (Daw et al., 2006; Badre et al., 2012; Raja Beharelle et al., 2015; Chakroun et al., 2020). Importantly, our initial hypothesis of attenuated FP activation in gamblers was not confirmed. Although FP and IPS effects of directed exploration were numerically smaller in gamblers (see Fig. 6), neither group difference was significant. However, the DCM analysis revealed some evidence for altered FP connectivity in gamblers (i.e., trend-level reductions in connectivity for FP-parietal cortex and FP-aIns interactions; see Fig. 7C).

Given that dopamine has been implicated in both the exploration/exploitation trade-off (Frank et al., 2009; Beeler, 2012; Kayser et al., 2015; Gardner et al., 2018; Chakroun et al., 2020) and GD (Voon et al., 2006; Boileau et al., 2014; Majuri et al., 2017; Mathar et al., 2018; Potenza, 2018; van Holst et al., 2018), we additionally conducted an exploratory analysis of subcortical correlates of directed exploration. The finding of reduced SN/VTA effects during exploration in gamblers resonates with a recent study that reported increased dopamine synthesis capacity in striatal regions in gamblers (van Holst et al., 2018), but see Potenza (2018) for a critical discussion. Given the reciprocal connectivity between striatum and SN/ VTA (Haber and Knutson, 2010), increased striatal-midbrain feedback inhibition might be one mechanism underlying this effect. However, small midbrain effects can be affected by cardiac or respiratory artifacts, which were not directly controlled in the present study.
Group membership could be decoded from the DCM coupling parameters with an accuracy of $73.2 \%$. This supports the idea that network interactions might contain additional information reflecting a participants' clinical status compared with univariate contrasts (Brodersen et al., 2014). However, we emphasize that the overall prediction accuracy, although significantly above chance-level based on permutation testing, is still too low for potential clinical applications. Higher accuracies could be achieved by larger training datasets (to reduce the noise induced by individual outliers). Also, more detailed network models might better reflect the underlying neural computations and thus yield higher predictive accuracy.

A number of limitations of the present study need to be acknowledged. First, it remains unclear whether reduced directed exploration constitutes a vulnerability factor or a consequence of continuous gambling. It would therefore be interesting to see whether these effects are tied to the clinical development of patients (e.g., to the escalation of gambling behavior or treatment effects) or whether they manifest as stable factors that increase the risk for the development of the disorder. Second, a comparison of the present results to patients with substance-use disorders would be of considerable interest (Morris et al., 2016), in particular given the overlap in terms of decision-making impairments. Third, tasks such as the observe-or-bet task (Blanchard and Gershman, 2018) or the horizon task (Wilson et al., 2014) might allow for a more clear-cut dissociation between directed and random exploration than the bandit task employed here. The reason is that the computational model assumes that value, 
perseveration and exploration bonus jointly affect action probabilities at the time of choice. Our classification of trials into exploitation, directed exploration and random exploration based on the fitted model therefore might not constitute as clear-cut segregation of the involved processes as in these other tasks. On the other hand, one advantage of the bandit task is that it assesses behavior as it unfolds over longer learning periods in a dynamic environment. As such, it might better resemble exploration behavior as it occurs in dynamic real-world settings. Finally, models with free random walk parameters showed convergence problems, such that these parameters (with the exception of $\sigma_{d}$ ) could only be estimated in a non-hierarchical fashion (Raja Beharelle et al., 2015; Chakroun et al., 2020). Allowing $\lambda$ or $\theta$ to vary still revealed a group difference in $\varphi$. Likely more data are required to reliably estimate these parameters, in particular in clinical samples such as the present one.

Impairments in reward-based learning, decision-making and cognitive control are hallmarks of GD (Wiehler and Peters, 2015; Clark et al., 2019). Here, we show using computational modeling that during reinforcement learning in volatile environments, gamblers' behavior is characterized by attenuated directed exploration rather than increased perseveration. Whether alterations in the exploration/exploitations trade-off extend to other tasks or environmental statistics, or could account for previous findings of reversal learning impairments in gamblers (de Ruiter et al., 2009; Boog et al., 2014) are interesting open question. Coupling parameters from a dynamic causal model of an explorationrelated network contained information predictive of clinical status, raising the possibility that such network interactions might be more diagnostic of this disorder compared with univariate effects. An exploratory analysis of subcortical exploration-related group differences revealed reduced activity in the SN/VTA in gamblers, complementing accumulating evidence for dopaminergic dysfunction associated with this disorder (Boileau et al., 2014; van Holst et al., 2018; van Timmeren et al., 2018; Clark et al., 2019; Kayser, 2019). Taken together, our findings highlight computational mechanisms underlying reinforcement learning in volatile environments in GD. In light of earlier results (Chakroun et al., 2020) this might be related to dopaminergic dysregulation.

\section{References}

Badre D, Doll BB, Long NM, Frank MJ (2012) Rostrolateral prefrontal cortex and individual differences in uncertainty-driven exploration. Neuron 73:595-607.

Balodis IM, Kober H, Worhunsky PD, Stevens MC, Pearlson GD, Potenza MN, Leyton M, Vezina P (2012) Attending to striatal ups and downs in addictions. Biol Psychiatry 72:e25-e26.

Bartra O, McGuire JT, Kable JW (2013) The valuation system: a coordinatebased meta-analysis of BOLD fMRI experiments examining neural correlates of subjective value. Neuroimage 76:412-427.

Beeler JA (2012) Thorndike's law 2.0: dopamine and the regulation of thrift. Front Neurosci 6:116.

Blanchard TC, Gershman SJ (2018) Pure correlates of exploration and exploitation in the human brain. Cogn Affect Behav Neurosci 18:117-126.

Boileau I, Payer D, Chugani B, Lobo DSS, Houle S, Wilson AA, Warsh J, Kish SJ, Zack M (2014) In vivo evidence for greater amphetamineinduced dopamine release in pathological gambling: a positron emission tomography study with [11C]-(+)-PHNO. Mol Psychiatry 19:13051313.

Boog M, Höppener P, vd, Wetering BJM, Goudriaan AE, Boog MC, Franken IHA (2014) Cognitive inflexibility in gamblers is primarily present in reward-related decision making. Front Hum Neurosci 8:569.

Boorman ED, Behrens TEJ, Woolrich MW, Rushworth MFS (2009) How green is the grass on the other side? Frontopolar cortex and the evidence in favor of alternative courses of action. Neuron 62:733-743.
Boorman ED, Behrens TE, Rushworth MF (2011) Counterfactual choice and learning in a neural network centered on human lateral frontopolar cortex. PLoS Biol 9:e1001093.

Brodersen KH, Deserno L, Schlagenhauf F, Lin Z, Penny WD, Buhmann JM, Stephan KE (2014) Dissecting psychiatric spectrum disorders by generative embedding. NeuroImage Clin 4:98-111.

Carpenter B, Gelman A, Hoffman MD, Lee D, Goodrich B, Betancourt M, Brubaker M, Guo J, Li P, Riddell A (2017) Stan: a probabilistic programming language. J Stat Softw 76:551-555.

Chakroun K, Mathar D, Wiehler A, Ganzer F, Peters J (2020) Dopaminergic modulation of the exploration/exploitation trade-off in human decisionmaking. Elife 9:e51260.

Chang CC, Lin CJ (2011) \{LIBSVM\}: a library for support vector machines. ACM Trans Intell Syst Technol 2:1-27:27.

Cinotti F, Fresno V, Aklil N, Coutureau E, Girard B, Marchand AR, Khamassi M (2019) Dopamine blockade impairs the exploration-exploitation trade-off in rats. Sci Rep 9:6770.

Clark L, Boileau I, Zack M (2019) Neuroimaging of reward mechanisms in gambling disorder: an integrative review. Mol Psychiatry 24:674-693.

Clithero JA, Rangel A (2014) Informatic parcellation of the network involved in the computation of subjective value. Soc Cogn Affect Neurosci 9:1289-1302.

Daw ND, O’Doherty JP, Dayan P, Seymour B, Dolan RJ (2006) Cortical substrates for exploratory decisions in humans. Nature 441:876-879.

Deichmann R, Gottfried JA, Hutton C, Turner R (2003) Optimized EPI for fMRI studies of the orbitofrontal cortex. Neuroimage 19:430-441.

de Ruiter MB, Veltman DJ, Goudriaan AE, Oosterlaan J, Sjoerds Z, van den Brink W (2009) Response perseveration and ventral prefrontal sensitivity to reward and punishment in male problem gamblers and smokers. Neuropsychopharmacology 34:1027-1038.

Driver-Dunckley E, Samanta J, Stacy M (2003) Pathological gambling associated with dopamine agonist therapy in Parkinson's disease. Neurology 61:422-423.

Frank MJ, Doll BB, Oas-Terpstra J, Moreno F (2009) Prefrontal and striatal dopaminergic genes predict individual differences in exploration and exploitation. Nat Neurosci 12:1062-1068.

Fujimoto A, Tsurumi K, Kawada R, Murao T, Takeuchi H, Murai T, Takahashi H (2017) Deficit of state-dependent risk attitude modulation in gambling disorder. Transl Psychiatry 7:e1085.

Gardner MPH, Schoenbaum G, Gershman SJ (2018) Rethinking dopamine as generalized prediction error. Proc Biol Sci 285:20181645.

Gelman A, Hwang J, Vehtari A (2014) Understanding predictive information criteria for Bayesian models. Stat Comput 24:997-1016.

Gershman SJ, Tzovaras BG (2018) Dopaminergic genes are associated with both directed and random exploration. Neuropsychologia 120:97-104.

Goudriaan AE, van den Brink W, van Holst RJ (2019) Gambling disorder. In: Gambling disorder (Heinz A, Romanczuk-SeiferthN, Potenza MN, eds). Cham: Springer International Publishing.

Haber S, Knutson B (2010) The reward circuit: linking primate anatomy and human imaging. Neuropsychopharmacology 35:4-26.

Heatherton TF, Kozlowski LT, Frecker RC, Fagerström K (1991) The Fagerström test for nicotine dependence: a revision of the Fagerström tolerance questionnaire. Br J Addict 86:1119-1127.

Kayser A (2019) Dopamine and gambling disorder: prospects for personalized treatment. Curr Addict Rep 6:65-74.

Kayser AS, Mitchell JM, Weinstein D, Frank MJ (2015) Dopamine, locus of control, and the exploration-exploitation tradeoff. Neuropsychopharmacology 40:454-462.

Kessler RC, Hwang I, LaBrie R, Petukhova M, Sampson NA, Winters KC, Shaffer HJ (2008) DSM-IV pathological gambling in the National Comorbidity Survey Replication. Psychol Med 38:1351-1360.

Lesieur HR, Blume SB (1987) The South Oaks Gambling Screen (SOGS): a new instrument for the identification of pathological gamblers. Am J Psychiatry 144:1184-1188.

Leyton M, Vezina P (2012) On cue: striatal ups and downs in addictions. Biol Psychiatry 72:10-12.

Ligneul R (2019) Sequential exploration in the IOWA gambling task: validation of a new computational model in a large dataset of young and old healthy participants. PLoS Comput Biol 15:e1006989.

Lim MSM, Jocham G, Hunt LT, Behrens TEJ, Rogers RD (2015) Impulsivity and predictive control are associated with suboptimal action-selection 
and action-value learning in regular gamblers. Int Gambl Stud 15:489505.

Lorains FK, Cowlishaw S, Thomas SA (2011) Prevalence of comorbid disorders in problem and pathological gambling: systematic review and metaanalysis of population surveys. Addiction 106:490-498.

Majuri J, Joutsa J, Johansson J, Voon V, Alakurtti K, Parkkola R, Lahti T, Alho H, Hirvonen J, Arponen E, Forsback S, Kaasinen V (2017) Dopamine and opioid neurotransmission in behavioral addictions: a comparative PET study in pathological gambling and binge eating. Neuropsychopharmacol 42:1169-1177.

Marsman M, Wagenmakers EJ (2017) Three insights from a Bayesian interpretation of the one-sided p value. Educ Psychol Meas 77:529-539.

Mathar D, Wiehler A, Chakroun K, Goltz D, Peters J (2018) A potential link between gambling addiction severity and central dopamine levels: evidence from spontaneous eye blink rates. Sci Rep 8:13371.

McClure SM, Berns GS, Montague PR (2003) Temporal prediction errors in a passive learning task activate human striatum. Neuron 38:339-346.

Miedl SF, Peters J, Büchel C (2012) Altered neural reward representations in pathological gamblers revealed by delay and probability discounting. Arch Gen Psychiatry 69:177-186.

Miedl SF, Büchel C, Peters J (2014) Cue-induced craving increases impulsivity via changes in striatal value signals in problem gamblers. J Neurosci 34:4750-4755.

Morris LS, Baek K, Kundu P, Harrison NA, Frank MJ, Voon V (2016) Biases in the explore-exploit tradeoff in addictions: the role of avoidance of uncertainty. Neuropsychopharmacology 41:940-948.

Osman A, Kopper BA, Barrios F, Gutierrez PM, Bagge CL (2004) Reliability and validity of the Beck depression inventory-II with adolescent psychiatric inpatients. Psychol Assess 16:120-132.

Payzan-Lenestour É, Bossaerts P (2012) Do not bet on the unknown versus try to find out more: estimation uncertainty and "unexpected uncertainty" both modulate exploration. Front Neurosci 6:150.

Pedersen ML, Frank MJ, Biele G (2017) The drift diffusion model as the choice rule in reinforcement learning. Psychon Bull Rev 24:1234-1251.

Penny WD, Stephan KE, Daunizeau J, Rosa MJ, Friston KJ, Schofield TM, Leff AP (2010) Comparing families of dynamic causal models. PLoS Comput Biol 6:e1000709.

Pessiglione M, Seymour B, Flandin G, Dolan RJ, Frith CD (2006) Dopaminedependent prediction errors underpin reward-seeking behaviour in humans. Nature 442:1042-1045.

Petry J (1996) Psychotherapie der Glücksspielsucht. Weinheim: Beltz, Psychologie-Verlag-Union.

Potenza MN (2018) Searching for replicable dopamine-related findings in gambling disorder. Biol Psychiatry 83:984-986.

Raja Beharelle A, Polanía R, Hare TA, Ruff CC (2015) Transcranial stimulation over frontopolar cortex elucidates the choice attributes and neural mechanisms used to resolve exploration-exploitation trade-offs. J Neurosci 35:14544-14556.

Raylu N, Oei TPS (2004) The gambling related cognitions scale (GRCS): development, confirmatory factor validation and psychometric properties. Addiction 99:757-769.
Reuter J, Raedler T, Rose M, Hand I, Gläscher J, Büchel C (2005) Pathological gambling is linked to reduced activation of the mesolimbic reward system. Nat Neurosci 8:147-148.

Saunders JB, Aasland OG, Babor TF, De la Fuente JR, Grant M (1993) Development of the alcohol use disorders identification test (AUDIT): WHO collaborative project on early detection of persons with harmful alcohol consumption - II. Addiction 88:791-804.

Schmitz N, Hartkamp N, Kiuse J, Franke GH, Reister G, Tress W (2000) The symptom check-list-90-R (SCL-90-R): a German validation study. Qual Life Res 9:185-193.

Schulz E, Gershman SJ (2019) The algorithmic architecture of exploration in the human brain. Curr Opin Neurobiol 55:7-14.

Speekenbrink M, Konstantinidis E (2015) Uncertainty and exploration in a restless bandit problem. Top Cogn Sci 7:351-367.

Stephan KE, Kasper L, Harrison LM, Daunizeau J, den Ouden HEM, Breakspear M, Friston KJ (2008) Nonlinear dynamic causal models for fMRI. Neuroimage 42:649-662.

Stephan KE, Penny WD, Daunizeau J, Moran RJ, Friston KJ (2009) Bayesian model selection for group studies. Neuroimage 46:1004-1017.

Sutton RS, Barto AG (1998) Introduction to reinforcement learning, Ed 1. Cambridge: MIT Press.

van Holst RJ, Veltman DJ, Büchel C, van den Brink W, Goudriaan AE (2012a) Distorted expectancy coding in problem gambling: is the addictive in the anticipation? Biol Psychiatry 71:741-748.

van Holst RJ, Veltman DJ, Van Den Brink W, Goudriaan AE (2012b) Right on cue? Striatal reactivity in problem gamblers. Biol Psychiatry 72:e23e24.

van Holst RJ, Sescousse G, Janssen LK, Janssen M, Berry AS, Jagust WJ, Cools R (2018) Increased striatal dopamine synthesis capacity in gambling addiction. Biol Psychiatry 83:1036-1043.

van Timmeren T, Daams JG, van Holst RJ, Goudriaan AE (2018) Compulsivity-related neurocognitive performance deficits in gambling disorder: a systematic review and meta-analysis. Neurosci Biobehav Rev 84:204-217.

Vehtari A, Gelman A, Gabry J (2017) Practical Bayesian model evaluation using leave-one-out cross-validation and WAIC. Stat Comput 27:14131432.

Voon V, Hassan K, Zurowski M, Duff-Canning S, de Souza M, Fox S, Lang AE, Miyasaki J (2006) Prospective prevalence of pathologic gambling and medication association in Parkinson disease. Neurology 66:1750-1752.

Watanabe S (2010) Asymptotic equivalence of Bayes cross validation and widely applicable information criterion in singular learning theory. J Mach Learn Res 11:3571-3594.

Wiehler A, Peters J (2015) Reward-based decision making in pathological gambling: the roles of risk and delay. Neurosci Res 90:3-14.

Wilson RC, Geana A, White JM, Ludvig EA, Cohen JD (2014) Humans use directed and random exploration to solve the explore-exploit dilemma. J Exp Psychol Gen 143:2074-2081.

Wilson RC, Bonawitz E, Costa VD, Ebitz RB (2021) Balancing exploration and exploitation with information and randomization. Curr Opin Behav Sci 38:49-56.

Zajkowski WK, Kossut M, Wilson RC (2017) A causal role for right frontopolar cortex in directed, but not random exploration. Elife 6:e27430. 